\title{
Model approximation for sound transmission from underwater structures in high-frequency range
}

\author{
Rui Zhang ${ }^{1,2}$, Desen Yang ${ }^{1,2}$, Shengguo Shi ${ }^{1,2,}$, and Boquan Yang $^{1}$ \\ ${ }^{1}$ College of Underwater Acoustic Engineering, Harbin Engineering University, Harbin 150001, China \\ ${ }^{2}$ Acoustic Science and Technology Laboratory, Harbin Engineering University, Harbin 150001, China
}

\begin{abstract}
Sound-insulation model provides a straightforward way to describe sound transmission behaviours of the thin-walled structures in engineering applications. The sound transmission characteristics depend on the parameters of incident wave, such as incident wave amplitude and incident angles. However, this model is limited when the sound source is located in an enclosed space (e.g., noise source in underwater cabins), because it is difficult to obtain incident angles especially in the high-frequency range. In this paper, we develop a simply analytical model that can effectively study the sound transmission from an enclosed shell with internal acoustic excitation. In order to extend the application of the sound-insulation model to a submerged shell, the structural vibration equation is firstly simplified to the plate vibration equation. Then, the sound pressure near the inner surface of the shell is decomposed into an expansion of orthogonal cavity eigenmodes, and each cavity mode is replaced by two pairs of incident plane waves. Finally, the acoustic transmission loss can be obtained by substituting the parameters of incident waves into the sound-insulation model. Numerical results show that the sound transmission for the fundamental cavity mode $(0,0,0)$ can be explained by the normal incidence in the sound-insulation model, while every other modes corresponds to a group of oblique incident plane waves whose incident angles decrease monotonically with the increase of frequency. In addition, it can be observed that the total reflection phenomenon in the sound-insulation model is consistent with the low radiation efficiency of the high order modes in the shell model.
\end{abstract}

\section{Introduction}

Thin cylindrical shells are the practical elements of various engineering structures such as pipes, space shuttles, car industry, aircraft and ship hulls[1-4]. The sound transmission of shell usually has a wide frequency range due to the variety of noise sources. Therefore, the establishment of different models are often required to make use of vibration characteristics of shell according to the range of the excitation frequency. The classical thin shell theories[5-7] provided the basis for studying the sound transmission problem of cylindrical structures. Fuller[8] presented the acoustic radiation characteristics of infinite cylindrical tube excited by an internal sound source, calculating shell mobility, far-field radiated pressure directivity and transmission loss. Based on the finite cylindrical shell models, Xia Pan[9-10] discussed in detail the parametric influence of shell on the radiated sound field by analytic and numerical methods, and made a comparison by analytic and finite element methods.

In addition, there are many literatures about sound transmission under external sound excitation. Smith[11] presented a method to estimate transmission of the sound energy through a thin, isotropic elastic cylindrical shell with the oblique plane wave excitation. J.H. Lee and J. $\operatorname{Kim}[12-13]$ studied the effect of periodically deployed stiffeners on the vibration response of cylindrical shells and experimentally demonstrated the qualitative trend from the well match between the theoretical and the measurement model. P. Oliazadeh et al.[14] investigated the acoustic behaviors of a double-walled and triplewalled thin circular cylindrical shell subjected to a plane incident wave. In his work, the prediction accuracy of Donnell's theory is better than that of Love's theory in the high frequency region.

All the above studies are based on the classical thin shell theory. However, as shell equations in which rotary kinetic energy and transverse shear are ignored have been employed, the analysis is gradually limited with the increase of frequency. Besides, the truncation of the decomposition series is also a computational restriction on the fluid-structure coupled equations. As is well known, the sound insulation theory[15] is applicable to sound transmission prediction for thin-walled structures. The classical work of this research is that of mass-airmass multilayer structures[16] are used for sound insulation in many fields. Fringuelino et al.[17] used a simplified approach to compute the transmission loss across multi-layer walls based on the characteristic impedance of each material layer. An experimental study by Tadeu et al.[18] showed the differences between the experimental and analytical results occurred at low frequencies, and mostly in the dips of insulation. To provide good absorption over a wide frequency range, Toyoda et al.[19-20] investigated improvements for the

Corresponding author: shishengguo@hrbeu.edu.cn 
sound insulation performance of multi-layer structures using a microperforated panel. Furthermore, $\mathrm{Mu}$ et al. [21] achieved higher transmission loss by using damping materials. In these cases, sound insulation theory has been developed and applied to semi-infinite sound insulation structures. However, the sound transmission prediction still depends on the angle and amplitude of incident wave, which contradicts an enclosed acoustic transmission model for the difficulty to obtain angle parameters.

In this paper, the sound insulation model is used to fast predict the sound transmission for a closed cylindrical shell as an alternative method in highfrequency range. Firstly, the study focuses on decomposing the sound pressure on the inner surface of the shell and simplifying the vibration equation of the shell. Then numerical simulations study the relationship between shell coupled equations and sound insulation equations and the results show the further applicability of the approximate model. Conclusions are presented in the last part.

\section{Sound transmission modelling}

A finite thin cylindrical shell with thickness $h$, radius $a$, length $L$ and bulk mass density $\rho_{\mathrm{s}}$ is considered for analysis. The cylindrical shell has two rigid end plates (to form a finite cylinder) which attach to two semiinfinite rigid baffles. Donnell's thin shell equations are used to describe the equations of motion of the cylindrical shell. The radial displacement of the shell, $w$ is coupled to the other two displacement components, namely, $u$ in the axial direction and $v$ in the circumferential direction. The equations are written as follow

$$
\begin{gathered}
\frac{\partial^{2} u}{\partial z^{2}}+\frac{1-\sigma}{2 a^{2}} \frac{\partial^{2} u}{\partial \phi^{2}}+\frac{1+\sigma}{2 a} \frac{\partial^{2} v}{\partial z \partial \phi}+\frac{\sigma}{a} \frac{\partial w}{\partial z}-\frac{\ddot{u}}{c_{p}^{2}}=0 \\
\frac{1+\sigma}{2 a} \frac{\partial^{2} u}{\partial z \partial \phi}+\frac{1-\sigma}{2} \frac{\partial^{2} v}{\partial z^{2}}+\frac{1}{a^{2}} \frac{\partial^{2} v}{\partial \phi^{2}}+\frac{1}{a^{2}} \frac{\partial w}{\partial \phi}-\frac{\ddot{v}}{c_{p}^{2}}=0 \\
\frac{\sigma}{a} \frac{\partial u}{\partial z}+\frac{1}{a^{2}} \frac{\partial v}{\partial \phi}+\frac{w}{a^{2}}+\frac{h^{2}}{12 a^{2}}\left(a^{2} \frac{\partial^{4} w}{\partial z^{4}}\right. \\
\left.+2 \frac{\partial^{4} w}{\partial z^{2} \partial \phi^{2}}+\frac{1}{a^{2}} \frac{\partial^{4} w}{\partial \phi^{4}}\right)+\frac{\ddot{w}}{c_{p}^{2}}=\frac{1-\sigma^{2}}{E h} p_{r}
\end{gathered}
$$

where $c_{\mathrm{p}}^{2}$ is defined by $c_{\mathrm{p}}^{2}=E / \rho_{\mathrm{s}}\left(1-\sigma^{2}\right) . \quad p_{\mathrm{r}}$ is the sound pressure on the surface of the cylindrical shell. $E$ is Young's modulus. Poisson's ratio is denoted by $\sigma$. Since the problem is linear, time dependence of all quantities can be assumed to be exp(-j $\omega t)$.

The displacement function of the shell simply supported on its end, which is satisfied with boundary conditions, can be expanded using modes as follows

$$
\left\{\begin{array}{l}
u(\theta, z) \\
v(\theta, z) \\
w(\theta, z)
\end{array}\right\}=\sum_{\alpha, m, n}\left\{\begin{array}{c}
U_{\alpha m n} \cos (n \theta-\alpha \pi / 2) \cos (m \pi z / L) \\
V_{\alpha m n} \sin (n \theta-\alpha \pi / 2) \sin (m \pi z / L) \\
W_{\alpha m n} \cos (n \theta-\alpha \pi / 2) \sin (m \pi z / L)
\end{array}\right\}
$$

where $U_{\alpha m n}, V_{\alpha m n}$ and $W_{\alpha m n}$ are the modal vectors of the corresponding simply supported shell with $n$ and $m$ being the circumferential and longitudinal order, respectively, and $\alpha=0$ (or 1 ) means the symmetric (or antisymmetric) mode.

Substituting Eqs. (4) into Eq. (3) gives the coefficient matrix of the spectral displacements

$$
\left[\begin{array}{lll}
S_{11} & S_{12} & S_{13} \\
S_{21} & S_{22} & S_{23} \\
S_{31} & S_{32} & S_{33}
\end{array}\right]\left[\begin{array}{l}
U_{n m} \\
V_{n m} \\
W_{n m}
\end{array}\right]=\left[\begin{array}{c}
0 \\
0 \\
F_{n m}
\end{array}\right]
$$

where

$$
\begin{aligned}
& S_{11}=-\Omega^{2}+k_{m}^{2} a^{2}+(1-\sigma) n^{2} / 2 \\
& S_{12}=(1+\sigma) n k_{m} a / 2 \\
& S_{13}=\sigma k_{m} a \\
& S_{22}=-\Omega^{2}+(1-\sigma) k_{m}^{2} a^{2} / 2+n^{2} \\
& S_{23}=n \\
& S_{33}=-\Omega^{2}+1+\beta^{2}\left(k_{m}^{2} a^{2}+n^{2}\right)^{2} \\
& S_{21}=S_{12}, S_{31}=S_{13}, S_{32}=S_{23} \\
& \Omega^{2}=\left(1-\sigma^{2}\right) \rho_{s} a^{3} \omega^{2} / E, \beta^{2}=h^{2} /\left(12 a^{2}\right)
\end{aligned}
$$

For a long cylindrical shell $(a / L<<1)$, Eq. (5) appears as a simplified form as follow

$$
\left[\begin{array}{cc}
-\Omega^{2}+n^{2} & n \\
n & -\Omega^{2}+1+\beta^{2} n^{4}
\end{array}\right]\left[\begin{array}{c}
V_{n m} \\
W_{n m}
\end{array}\right]=\left[\begin{array}{c}
0 \\
F_{n m}
\end{array}\right]
$$

As the frequency increases, the vibration of the shell is dominated by the high order mode. And then, the axial displacement, circumferential displacement and radial displacement of the shell vibration equation are gradually decoupled. The vibration equation of cylindrical shells degenerates into the following form

$$
\frac{E h^{3}}{12\left(1-\sigma^{2}\right)} \frac{\partial^{4} w}{\partial s^{4}}+\rho h \ddot{w}=p_{r}
$$

In this case, the vibration equation of the shell is reduced to the vibration equation of the plate. Furthermore, the sound transmission loss (TL) of cylindrical shells can be obtained by using the soundinsulation theory of plates as follows

$$
T=\frac{2 Z_{0}^{(2)}\left(Z_{n p}-Z\right) e^{-j k_{2} h \cos \theta_{2}}}{Z Z_{n p}+4 Z_{0}^{(1)} Z_{0}^{(2)}+\left(Z_{0}^{(1)}+Z_{0}^{(2)}\right)\left(Z+Z_{n p}\right)}
$$

where $Z_{0}^{(1)}=\rho_{1} c_{1} / \cos \theta_{1}, Z_{0}^{(2)}=\rho_{2} c_{2} / \cos \theta_{2}$,

$$
\begin{aligned}
& Z=j \omega \rho_{s} h\left(1-c_{p}^{4} / c^{4} \cdot \sin ^{4} \theta\right), \\
& Z_{n p}=4 i E(1-\sigma) /[\omega h(1-2 \sigma)(1+\sigma)] .
\end{aligned}
$$

In order to obtain the parameters of incident wave in Eq. (8), the sound pressure near the inner surface of the shell needs to be decomposed into traveling wave form. The incident waves in a sound-insulation model usually have the expression forms as follow

$$
p_{i}(\omega, x)=p_{0} e^{j k_{0} l \cdot \sin \theta_{i}}
$$


where $p_{0}, \theta_{i}$ are the pressure amplitude and angle of the incident wave, respectively.

Different from the incident plane wave in the semiinfinite space, the sound field in an enclosed cylindrical shell is a reverberation sound field. The sound pressure of the inner surface of the cylindrical shell can be decomposed using an orthogonal expansion in terms of the hard-walled cavity modes

$$
\begin{aligned}
p(a, \theta, z) & =\sum_{\tilde{n}} \frac{\phi_{\tilde{n}}(a, \theta, z) \phi_{\tilde{n}}\left(r_{k}\right)}{\phi_{\tilde{n}}(r) \phi_{\tilde{n}}^{*}(r) d V \cdot\left(k_{\tilde{n}}^{2}-k^{2}\right)} \\
& =\sum_{n, m} A_{n m} \cos (n \theta) \cos (m \pi z / \mathrm{L})
\end{aligned}
$$

Eq.(10) shows that the sound pressure near the inner surface of the shell is represented by standing waves. Using the Euler equation, the Eq.(10) can be decomposed into as follows decomposed into as follows

$$
\begin{aligned}
& p(a, \theta, z)=\frac{1}{4} \sum_{n, m} A_{n m}\left(e^{-j(n \theta+m \pi z / \mathrm{L})}+\right. \\
& \left.e^{j(n \theta+m \pi z / \mathrm{L})}+e^{-j(n \theta-m \pi z / \mathrm{L})}+e^{j(n \theta-m \pi z / \mathrm{L})}\right)
\end{aligned}
$$

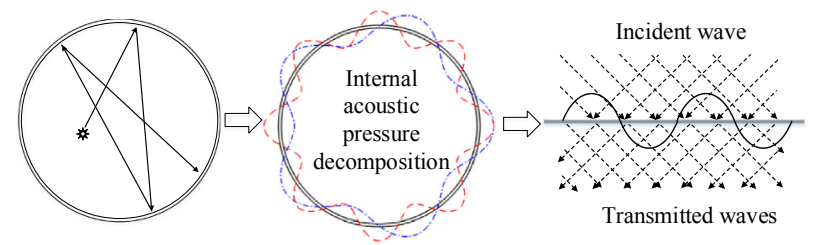

Fig. 1. Approximate procedure of sound transmission from cylindrical shell model to plate model.

Compared with Eq. (9), it should be observed that each cavity mode is replace by two pairs of incident plane waves with the opposite incident angle, as shown in Fig.1. Considering that the Eq. (9) is one-dimensional, the conditions for the corresponding phenomenon $(m=0$, or $n=0)$ in cylindrical shells could be adopted in Eq. (11). Thus, the incident angle in the shell model can be obtained as follows

$$
\begin{gathered}
\theta=\arcsin \left(n / k_{0} a\right) \\
\text { or } \theta=\arcsin \left(m \pi / k_{0} L\right)
\end{gathered}
$$

Compared with Eq. (9), when $m, n=0$, the expression form of Eq.(11) is similar to the normal incidence of plate model. For other modes, the correspondences are more likely to the oblique incidence of plane waves, and the incident angles vary with the frequency. Therefore, it can be seen that the sound transmission of each cavity mode in the shell model can be predicted by studying the sound-insulation model of plate with continuous incidence angles.

In the special case where the incident angle satisfies the condition of $\sin \theta_{i}>c_{1} / c_{2}$, total internal reflection occurs in the plate model. Under this condition, the energy of incident wave cannot be effectively transferred from interior of the shell to the external fluid. The conditions for the corresponding phenomenon $(m=0$, or $n=0$ ) in cylindrical shells should be satisfied as follow

$$
f_{c}=\frac{c_{2}^{2}}{2 a c_{1}} n \text { or } f_{c}=\frac{c_{2}^{2}}{2 L c_{1}} m
$$

where $f_{c}$ is the critical frequency of incident wave above which the energy can be effectively transferred. Eq. (13) shows that the high order mode has the low radiation efficiency in low-frequency range, which is consistent with the modal characteristics of the radiation impedance for cylindrical shells.

\section{Numerical analysis}

In order to analyse the sound transmission characteristics of the approximate model, a finite cylindrical shell is taken as an example whose parameters are given as follows: radius $a=1 \mathrm{~m}$, length $L=10 \mathrm{~m}$ and thickness $h=0.01 \mathrm{~m}$, Young's modulus $E=2.1 \times 10^{11} \mathrm{~N} / \mathrm{m}^{2}$, Poisson ratio $\sigma=0.3$, material density of the shell $\rho_{\mathrm{s}}=7800 \mathrm{~kg} / \mathrm{m}^{3}$. The fluid parameters include air medium inside the shell with material density $\rho_{1}=1.29 \mathrm{~kg} / \mathrm{m}^{3}$, sound velocity $c_{1}=343 \mathrm{~m} / \mathrm{s}$ and water medium outside the shell with material density $\rho_{2}=1000 \mathrm{~kg} / \mathrm{m}^{3}$, sound velocity $c_{2}=1500 \mathrm{~m} / \mathrm{s}$. The TL is defined as the ratio of the incoming and transmitted sound powers of the radiating plate.
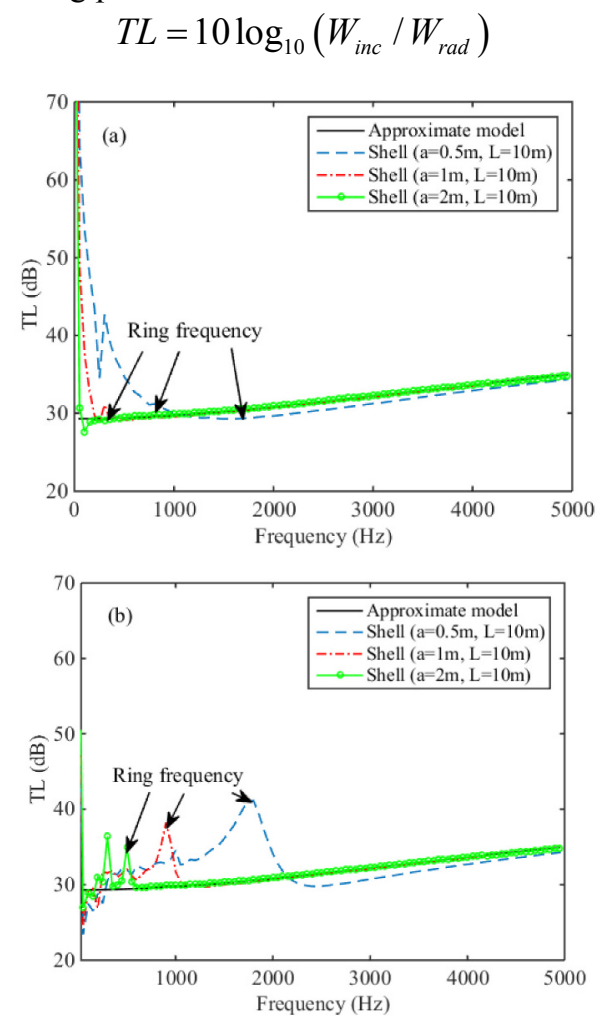

Fig. 2. Effect of shell radius on acoustic transmission (a) cavity mode $(0,0)$ (normal incidence); (b) cavity mode $(1,0)$ (oblique incidence).

The validity of the model approximation is related to the geometric size of the shell and acoustic wavelength. Ring frequency is an important parameter for the transition from shell to plate, which is given by $f_{r}=\sqrt{E / \rho\left(1-\sigma^{2}\right)} /(2 \pi a)$. The results are shown in Fig.2 
which indicates good agreement between the present results and those obtained by shell model above the ring frequency. Obviously, the cylindrical shell with larger radius agrees better with the approximate models. Besides, under the normal incidence, the sound transmission characteristics of shells and plates can be consistent at lower frequencies.
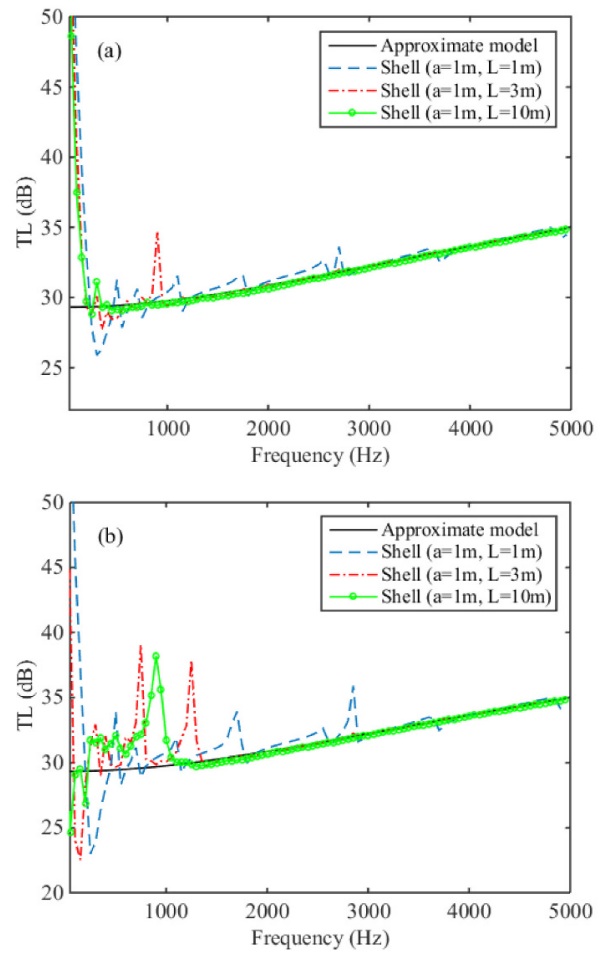

Fig. 3. Effect of shell length on acoustic transmission (a) cavity mode $(0,0)$ (normal incidence); (b) cavity mode $(1,0)$ (oblique incidence).
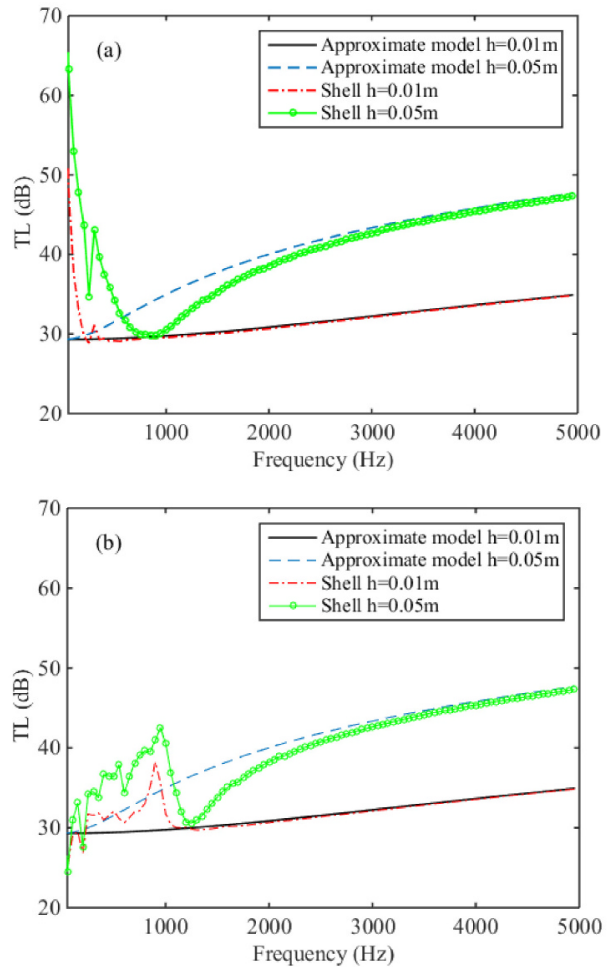

Fig. 4. Effect of shell thickness on acoustic transmission (a) cavity mode $(0,0)$ (normal incidence); (b) cavity mode $(1,0)$ (oblique incidence).
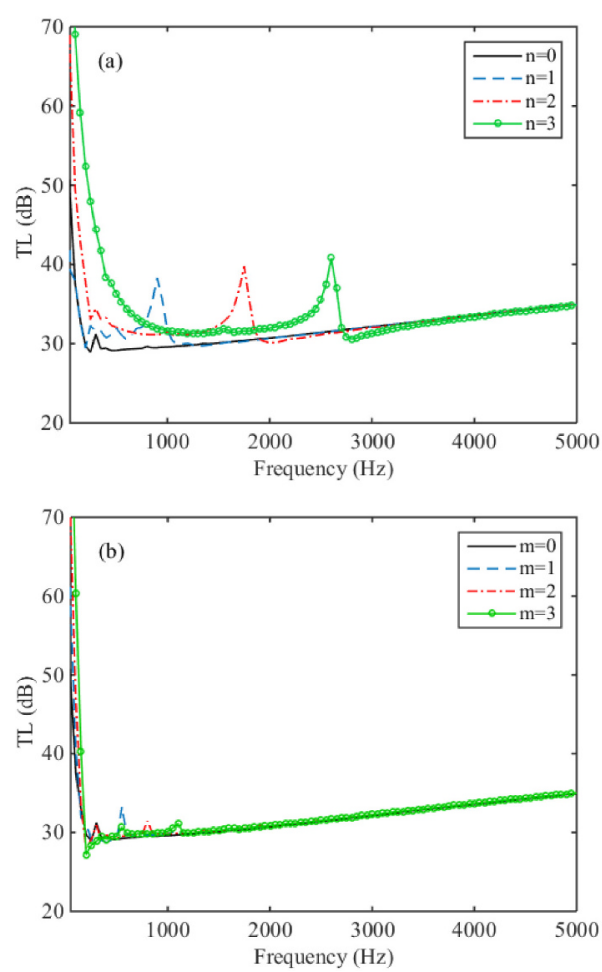

Fig. 5. Sound transmission losses of different cavity modes (a) $\mathrm{m}=0, \mathrm{n}=0 \sim 3$; (b) $\mathrm{n}=0, \mathrm{~m}=0 \sim 3$.
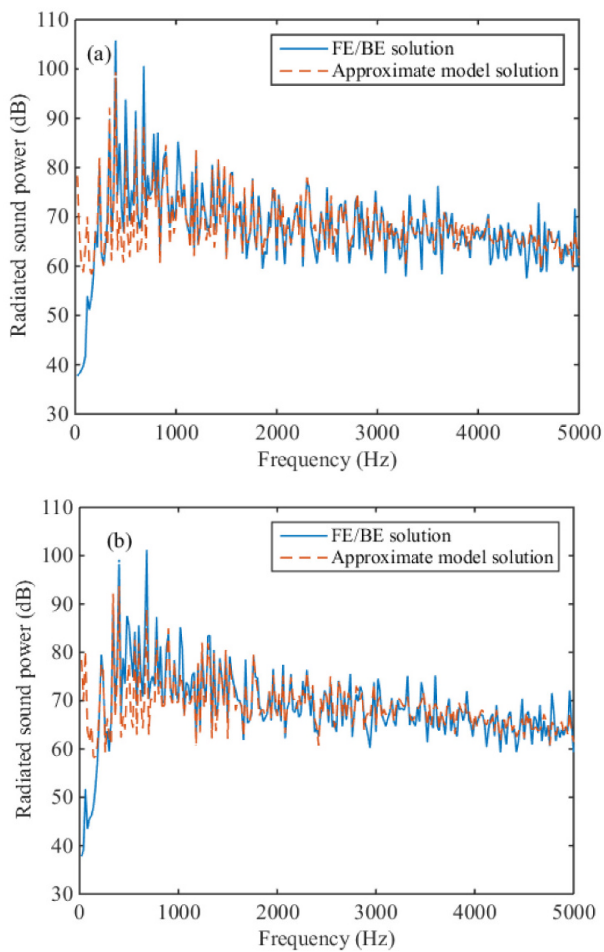

Fig. 6. Prediction of sound transmission excited by a monopole source excitation (a) with central excitation; (b) with end excitation.

Fig. 3 shows a comparison of the shells with different lengths. It can be seen that the length of the shell has little effect on TL, but the longer cylindrical shells agree better with the approximate models at lower frequencies. The TL under oblique incidence is relatively violent at low frequencies. The peak of the TL curves is caused by the structural resonance of the shell. With the frequency increasing, the TL curves of different shells are well 
coincident except for the structural resonance frequencies.

The thickness of the structure is directly related to the mass matrix and stiffness matrix of the structure, thus affects its sound transmission ability. Fig.4 shows a comparison of the TL with different thickness. It could be found that the thickness of the shell has a dominant influence on TL. Meanwhile, the prediction errors of the thicker shell are larger, and the effective frequency range of the model is relatively poor. In other aspects, the frequency characteristics of sound transmission are similar with those obtained before.

Further analysis is made to compare TL of the different cavity modes in Fig.5. It can be observed that the TL of different cavity modes is basically consistent with the normal incidence in high-frequency range, although there are some deviations near the resonant frequencies of the shell. Considering the principle of superposition applied to the sound-insulation theory, Fig.5 indicates that the trend of TL curves can ignore the effect of oblique incidence, thus the trend prediction of sound transmission can only take into account the normal incidence.

The sound transmission prediction of cylindrical shells due to acoustic excitation is calculated by the approximate model. A relatively realistic comparison model is established by using coupled FE/BE method. Fig. 6 presents the prediction of the sound transmission excited by acoustic excitation at different positions. Below the ring frequency, it is invalid for the prediction based on sound insulation principle. The results in the high-frequency region indicate that the approximate model can be used to predict the general trend of sound transmission in engineering.

\section{Conclusions}

In this study, the sound-insulation model has been extended to predict the sound transmission of a submerged cylindrical shell. By simplifying the vibration equation, the shell model satisfies the applicable conditions of sound-insulation model in the highfrequency region. The internal pressure of the cylindrical shell is decomposed to obtain the incident parameters in the sound-insulation model. Thus, the approximate model is established to predict the TL of a submerged cylindrical shell.

The validity of the approximate model is related to the geometric size of the shell. The radius of the shell directly determines the effective frequency range of the approximate model, while the longer cylindrical shell can slightly improve the prediction accuracy. And the numerical results show that thickness of the shell has a dominant influence on TL. Besides, the sound transmission characteristics of a cylindrical shell are analysed and compared with the FE/BE solution. Generally, the approximate model can preliminarily predict the sound transmission for a submerged cylindrical shell in high-frequency range.
1. S. K. Jha, J. J. Catherines, J. Sound Vib. 71, 523 (1980)

2. A. J. Bullmore, P. A. Nelson, S. J. Elliott, J. Sound Vib. 140, 191 (1990)

3. K. Daneshjou, A. Nouri, R. Talebitooti, J. Arch. Appl. Mech. 77, 363 (2007)

4. Bo Q, Zhu BL, Noise Vib. Contr. 6, 44 (2002)

5. M. C. Junger, J. App. Mech. 5, 35 (1954)

6. J. Zhou, A. Bhaskar, X. Zhang. J. Sound Vib. 333, 1972 (2014)

7. M. Amabili, Nonlinear Vibrations and Stability of Shells and Plates (Cambridge University, New York, 2008)

8. C. R. Fuller, J. Sound Vib. 109, 2 (1986)

9. X. Pan, I. MacGillivray, Y. Tso, H. Peters, Proc Acoustics, 2013, Australia, Victor Harbor

10. X. Pan, Y.Tso, J. Forrest and H. Peters, 2014 Inter. Noise, 2014, Australia, Melbourne

11. P. W. Smith, J. Acoust. Soc. Am. 29, 721 (1957)

12. J. H. Lee, J. Kim, J. Sound Vib. 251, 3 (2002)

13. J. H. Lee, J. Kim, Appl. Acoust. 64, 611 (2003)

14. P. Oliazadeh, A. Farshidianfar, J. Sound Vib. 389, 276 (2017)

15. A. Warnock, W. Fasold, Encyclopedia of acoustic (Wiley-Interscience, New York, 1997)

16. A. London, J. Acoust. Soc. Am. 22, 2 (1950)

17. M. Fringuelino, C. Guglielmone, Appl. Acoust. 29, 275 (2000)

18. A. Tadeu, J. Antonio, D. Mateus, Appl. Acoust. 65, 15 (2004)

19. M. Toyoda, D. Takahasi, J. Acoust. Soc. Am. 124, 6 (2008)

20. R. L. Mu, M. Toyoda, D. Takahashi, Appl. Acoust. 72, 849 (2011)

21. R. L. Mu, M. Toyoda, D. Takahashi, Noise Control Engr. J. 60, 4 (2012)

\section{References}

